\title{
Gesualdo Bufalino. Io è gli altri
}

Gesualdo Bufalino. I Is the Others

Gesualdo Bufalino. Je est les autres

\section{Gino Ruozzi}

\section{OpenEdition \\ Journals}

Edizione digitale

URL: http://journals.openedition.org/cei/6877

DOI: 10.4000/cei.6877

ISSN: 2260-779X

\section{Editore}

UGA Éditions/Université Grenoble Alpes

\section{Edizione cartacea}

ISBN: 978-2-37747-173-7

ISSN: $1770-9571$

\section{Notizia bibliografica digitale}

Gino Ruozzi, « Gesualdo Bufalino. Io è gli altri », Cahiers d'études italiennes [Online], 30 | 2020, Messo online il 01 mars 2020, consultato il 06 mars 2020. URL : http://journals.openedition.org/cei/6877 DOI : https://doi.org/10.4000/cei.6877

Questo documento è stato generato automaticamente il 6 marzo 2020

(C) ELLUG 


\title{
Gesualdo Bufalino. Io è gli altri
}

\author{
Gesualdo Bufalino. I Is the Others \\ Gesualdo Bufalino. Je est les autres
}

Gino Ruozzi

1 La citazione è uno dei punti forza e di fascino della scrittura e della personalità di Gesualdo Bufalino. È una sagra di luci, di memorie e rimandi a catena, di libere associazioni creative, che spaziano dalla letteratura alla musica all'arte alla geografia alla storia: ogni cosa è assorbita e trasformata nell'opera di Bufalino, che l'accoglie con piacere e allegria, con la gioia della potenza evocatrice e inventiva della letteratura.

2 In Bufalino la citazione è anche una precisa struttura stilistica e retorica, specialmente nei libri di aforismi dove acquista il valore di un modello specifico di aforisma: l'aforisma citazione.

3 Di questo particolare aspetto di reciprocità tra citazione e aforisma avevo trattato trent'anni fa in un saggio ${ }^{1}$ poi rivisto e ampliato ${ }^{2}$. Il principale oggetto di analisi era la raccolta di aforismi Il malpensante ${ }^{3}$, che per me ha avuto un'importanza fondamentale, poiché ha segnato il passaggio dallo studio degli aforismi, che avevo già iniziato con la tesi di laurea sui Pensieri diversi di Francesco Algarotti e la conseguente edizione moderna dell'opera (Angeli, 1987), alla passione per il genere e quindi a una scelta ancora più motivata sul piano della ricerca. Devo quindi a Bufalino e al suo Malpensante il principale percorso dei miei studi e una passione che dura tuttora con costante interesse.

4 Anche nei Pensieri diversi di Algarotti, pubblicati postumi a Pisa nel 1765 (l'anno dopo la morte dell'autore) la citazione riveste un ruolo fondamentale, come d'altronde nella letteratura umanistica e sapienziale da Erasmo e Montaigne in poi. La citazione è spesso determinante nella costruzione del pensiero e ha pertanto non solo un ruolo ornamentale ma produttivo. Sul piano retorico essa ha una funzione nodale anche in grandi opere memorialistiche e narrative quali l'Histoire da ma vie di Giacomo Casanova, non a caso bibliotecario a Dux in Boemia proprio negli anni della sua stesura.

5 Nella letteratura aforistica la citazione non è necessaria ma, paradossalmente, è la letteratura aforistica ad essere oggetto di citazione, grazie alla propria intrinseca 
brevità. Così come ogni citazione può diventare, per le stesse ragioni di concisione, aforisma. La tradizione medievale dei fiori di sentenze, degli ammaestramenti degli antichi, dei motti e degli adagi ne è la dimostrazione. Si tratta di aforismi per estrapolazione, che è il motivo per cui Shakespeare è lo scrittore di aforismi più citato al mondo senza avere scritto opere specifiche e intenzionali di aforismi.

6 Preciso la frase d'apertura del paragrafo precedente. Nella letteratura aforistica intenzionale la citazione non è necessaria. L'esempio più celebre è La Rochefoucauld, che non usa citazioni dirette e traduce la propria cultura esclusivamente attraverso la propria voce, così come fa Oscar Wilde. Nietzsche si comporta diversamente, così come avevano fatto anche Guicciardini nei Ricordi e Montaigne negli Essais, dialogando con la cultura, per lo più nella prospettiva del commento. Bufalino fa invece entrare in modo massiccio la cultura negli aforismi (e in tutti i libri), utilizzando la citazione sia per commenti sia come voce propria, in un'ottica polifonica. Nei libri di aforismi di Bufalino le voci chiamate in campo sono tantissime e assomigliano agli ingressi dei cavalieri nei poemi cavallereschi: danno mobilità al testo, introducono decine di punti di vista, dialogano in presenza e a distanza, compongono uno spettacolare gioco di indizi e di identificazioni. La quantità di citazioni può piacere ma può anche infastidire. Elias Canetti nei quaderni di appunti della Provincia dell'uomo scrive che «In Montaigne mi disturba spesso il grasso delle citazioni» ${ }^{4}$.

7 Per Bufalino la citazione è un grande piacere, come afferma in questo aforisma del Malpensante:

Mi attira il gioco del collage, l'intarsio di passi antichi dentro contesti moderni, un puledro delle Georgiche messo a scalpitare in una campagna normanna, un'isba freddolosa di nevi campita contro un cielo d'Estremadura. Sogno un libro dove storia e tempo si alleino a confondere in guazzabuglio le due tre evidenze in cui credo $^{5}$.

8 A Bufalino piace mescolare, intrecciare, viaggiare con la fantasia, come Pascoli in Romagna "galoppare immobilmente» sulla scia dell'immaginazione letteraria, ambedue attratti dal «sognato alone» delle fantasticazioni, nuovi cavalieri che si immedesimano negli antichi cavalieri di Boiardo e di Ariosto.

9 Nel Malpensante le citazioni aprono e chiudono il volume, sia all'esterno che all'interno. All'esterno perché in copertina titolo e sottotitolo corrispondono ad aperte citazioni di Leopardi: «malpensante» dai Paralipomeni della Batracomiomachia (V 24): «Dirovvi il parer mio da mal pensante / Qual da non molto in qua son divenuto», in perfetta sintonia con il primo dei Pensieri, in cui Leopardi dichiara di avere cambiato parere sugli uomini costretto dalla violenza dell'esperienza e conclude «che il mondo è una lega di birbanti contro gli uomini da bene e di vili contro i generosi». Il sottotitolo Lunario dell'anno che fu è ispirato all'operetta morale Dialogo di un venditore d'almanacchi $e$ di un passeggere. Leopardi è insegna emblematica, segno di un'affinità sostanziale, pungente e polemica. Tuttavia in Bufalino la disperazione, con ironica ammissione, è forse più detta che provata («Grido, è vero, ma a fior di labbro» $\left.{ }^{6}\right)$. Malpensante rimanda inoltre a una tradizione aforistica italiana consolidata: ai Pensieri d'un malpensante di Domenico Giuliotti (Vallecchi, 1936; i primi apparvero sul «Frontespizio» nel 1929), seguiti dai Nuovi pensieri d'un malpensante del 1947; e ai Pensieri di un libertino di Arrigo Cajumi, pubblicati da Longanesi in versione ridotta nello stesso 1947 e da Einaudi in edizione completa nel 1950 (la terza sezione dell'opera, datata 1937, è intitolata Il malpensante). Malpensanti di sponde ideali diverse: Giuliotti intransigente cattolico e già «omo salvatico» con Papini nel 1923 (Dizionario dell'omo salvatico, Vallecchi, lettere A 
e B); Cajumi esponente di uno schietto e tagliente laicismo liberale piemontese. Critico acuto, «assiduo lettore» e "scrittore aforistico e frammentario» (Santoli), Cajumi attribuisce grande importanza all'arte del saper citare: «Citar molto è una delle grandi necessità e qualità della critica: il valore del giudice si rivela dai testi ch'egli sa mettere in luce. Sainte-Beuve anche in questo fu un maestro incomparabile» ${ }^{7}$. Il discorso illumina Bufalino scrittore di aforismi e soprattutto autore di elzeviri.

La citazione o meglio le citazioni sono in primo piano anche nella quarta di copertina del Malpensante, testo d'autore e parte integrante dell'opera stessa, spumeggiante esibizione di cultura aforistica e di affinità elettive, ultima effettiva pagina del testo:

Malpensante è chi pensa male, tecnicamente parlando. Ma è, soprattutto, chi pensa male e ne accarezza i nodi dentro di sé, senza risolversi a tagliarli con un'energica scure. Di entrambi i significati sostiene d'essersi ricordato Gesualdo Bufalino nell'intitolare la presente raccolta di aforismi, note azzurre, fusées, greguerías, obiter dicta, goliarderie, malumori e umori, disposti a mo' di barbanera retrospettivi e offerti al passeggero, come si usava una volta. Uno zibaldone (o anche un diario travestito da libro sapienziale, un'opera dei pupi indecisa fra divertimento e passione) assai voluminoso in origine, ma da cui l'autore ha estratto solo le schegge che gli apparissero anticipi o riassunti delle sue più tenaci ossessioni. Non già, figurarsi, per inseguire modelli altissimi o alti, da Leopardi a Baudelaire, da Karl Kraus a Bierce, a Lec, a Flaiano; ma speranzoso che qualche lampo, sebbene livido e storto, si sprigioni dalle sue carte e induca un salutare sconcerto nel benpensante lettore.

11 È un testo bellissimo, tra i più sintetici e affascinanti di Bufalino. C'è una biblioteca in pillole. Ovviamente Leopardi: dal «malpensante» d'apertura all'opposto «benpensante lettore» di chiusura, che rinvia pure all'«hypocrite lecteur» dell'avviso al lettore dei Fiori del male di Baudelaire. Ancora Leopardi con gli almanacchi «offerti al passeggero», lo «zibaldone» e il nome proprio ad avviare la serie dei «modelli altissimi o alti» seguito da Baudelaire, autore di fusèes elencate tra i nomi metaforici di aforismi in compagnia delle «note azzurre» di Carlo Dossi, delle greguerías di Ramón Gómez de la Serna, degli obiter dicta di Jonathan Swift.

12 Le greguerías di Gómez de la Serna sono state una passione di Bufalino che le ha lette con ammirazione, tradotte, scelte e presentate con il titolo di Sghiribizzi (l'opera è uscita pochi mesi dopo la morte nel gennaio 1997 nei tascabili Bompiani). Nella premessa egli dichiara il proprio trasporto:

Le volte (le parecchie volte) che mi succede di star male e di chiedere a un libro non un ennesimo contagio di complice desolazione ma l'inganno di un'euforia, la mano cerca negli scaffali pagine liete: il grande Feydeau, il grande Woodhouse... Se danno poco profitto e il sollievo ritarda, la risorsa suprema è tradurre qualcuna fra le innumerevoli (dodici mila? quindici mila?) greguerías di Ramón Gómez de la Serna.

13 La predilezione è massima; in Gómez de la Serna Bufalino ritrova proprie ossessioni e vi riconosce un comune «decisivo non-sense del suo destino, enigma senza specchio, dietro cui preme l'impaziente fiumana del nulla». Sull'«arbitraria» scelta di tradurre con sghiribizzi l'originale greguerías Bufalino si affida al dizionario di Fanfani per il quale la parola «vale "capriccio, pensiero fantastico e strano". Forse il meno indegno supplente del termine originario, non fosse che per la vaga (meno che vaga) assonanza» ${ }^{8}$. Credo tuttavia che abbia avuto peso anche il ricordo del «ghiribizzo» con il quale Machiavelli parla del Principe nella famosa lettera a Francesco Vettori del 10 dicembre 1513 (senza dimenticare i Ghiribizzi novecenteschi di Roberto Ridolfi stampati da Vallecchi nel 1968). 
14 Altro termine significativo è «schegge», che rinvia alle Schegge di Giovanni Papini pubblicate già su «Lacerba» nel 1913 e infine sul «Corriere della Sera» nel 1954-1956, prima stampate da Vallecchi nei volumi Laspia del mondo. "Schegge di poesia e di esperienza» (1955) e Le felicità dell'infelice. Le ultime «schegge» (1956), poi unite in volume unico ancora da Vallecchi nel 1971. Nell'introduzione a Le felicità dell'infelice Papini spiega perché ha «dato il nome di schegge a questi frammenti di pensieri, a questi spunti di poesia, a queste tessere di capricci». Tra le varie ragioni, tra cui quella che «altre denominazioni erano già in uso», la principale è che «paragonando l'arte mia a quella dello statuario mi è venuto fatto di vedermi dinanzi ai piedi quelle schegge o scaglie di marmo tolte via via dal blocco per trarne fuori una libera, vera immagine» ${ }^{9}$. Con più leggerezza Bufalino riprende fuori di metafora l'idea di uno zibaldone «assai voluminoso in origine, ma da cui l'autore ha estratto solo le schegge che gli apparissero anticipi o riassunti delle sue più tenaci ossessioni». Di Papini si può ancora richiamare la Confessione preliminare a Il sacco dell'orco (Vallecchi, 1933), in cui figurano nomi e modi impiegati anche da Bufalino:

So benissimo che a raccolte di pensieri aforismi bizzarrie come la presente non si fa in Italia, di solito, buon viso. Si accettano, semmai, quando son postume - lo Zibaldone del Leopardi, le Note azzurre del Dossi e qualche altra - ma quelle dei viventi di rado piacciono: uniche eccezioni, credo, quelle di due artisti: il Giornale di bordo di Ardengo Soffici e il Pittore volante di Anselmo Bucci. E siccome non ho intenzione di morire prima del tempo per ingraziarmi i lettori arrischio anch'io, incoraggiato dagli ultimi esempi, questa silloge di frammenti. Non sono, per me, spurghi e resti messi dapparte nella fabbricazione delle altre opere. E se anche fossero trucioli avrebbero lo loro utilità, come quelli del legno, e una loro giustificazione. Ma per me queste malinconie, queste osservazioni sparse, questi appunti ironici o satirici, questi giudizi epigrafici, questi raccostamenti impensati o ridevoli, queste notazioni fuggitive, queste malignità benevole e queste amare lepidezze, sono operette a sé, colla propria ragion d'essere, e non sfornite, mi sembra, di valore suggestivo o documentario.

Bufalino poteva leggere queste pagine di Papini nei volumi Prose morali (settimo volume di Tutte le opere di Papini, Mondadori, 1959) e Pagine scelte (a cura di Giuseppe Cantamessa, Edizioni Scolastiche Mondadori, 1962), entrambi presenti nella sua biblioteca. Di Papini è famoso il passaggio di Un uomo finito (Libreria della «Voce», 1913) in cui afferma che «scontento ed eccitato, avido e schivo, scaricavo il mio sdegno in aforismi corrosivi, in sfoghi lirici e mordaci a somiglianza di quelli di Nietzsche» ${ }^{10}$.

I nomi di Leopardi e di Dossi sono con ogni evidenza cardini della tradizione italiana, ai quali Papini aggiunge i contemporanei Soffici e Bucci. Soffici fu compagno di imprese editoriali tra cui la rivista «Lacerba» (1913-1915) che spicca per le sue predilezioni aforistiche (il Giornale di bordo e i Razzi di Soffici, i Frammenti e lo Zibaldone di Italo Tavolato, Spazzatura di Palazzeschi, le Schegge dello stesso Papini). Bucci fu tra i protagonisti del Novecento pittorico italiano (con Malerba, Sironi, Oppi, Funi, Dudreville, Marussig e la promozione di Margherita Sarfatti); nel 1929 pubblicò da Ceschina il volume di aforismi Il pittore volante, con cui l'anno seguente vinse il primo premio Viareggio (condiviso con Lorenzo Viani).

17 Va comunque ricordato che sia lo Zibaldone di pensieri di Leopardi sia le Note azzurre di Dossi sono acquisizioni novecentesche. Lo Zibaldone di pensieri fu pubblicato postumo nel 1898 nel centenario della nascita di Leopardi col titolo Pensieri di varia filosofia e di bella letteratura a cura di una commissione ministeriale presieduta da Carducci (7 volumi, Le Monnier, 1898-1900). Il titolo di Zibaldone, desunto dall'«Indice del mio 
Zibaldone di Pensieri cominciato agli undici di luglio del 1827, in Firenze», venne usato da Ildebrando Della Giovanna nel Saggio dello «Zibaldone» incluso nell'edizione delle Prose morali di Leopardi pubblicata da Sansoni nel 1899. Le Note azzurre di Carlo Dossi, scritte su quaderni di copertina azzurra tra il 1870 e il 1907, furono pubblicate postume nel 1912 da Treves, in una prima edizione parziale curata dalla moglie Carlotta Borsani. La raccolta completa fu curata da Dante Isella nel 1956 per l'editore Ricciardi e poi riproposta in ampie selezioni da Adelphi $(1964,1988,2010$ : Bufalino possedeva quella del 1988; di data antecedente al Malpensante aveva il volume Garzanti Le più belle pagine scelte da Piero Nardi, 1940). La pubblicazione postuma di parecchie opere di aforismi, a cominciare da Guicciardini, è un altro elemento sintonico con Bufalino autore 'postumo' (come del resto tutta l'umanità: «Dio è morto creandoci, noi siamo un'opera postuma» $\left.{ }^{11}\right)$.

Nelle prefazioni di Papini risalta un altro elemento comune: l'enumerazione dei nomi con cui vengono chiamate le scritture aforistiche, per lo più in chiave metaforica. Si veda il termine «trucioli» che allude ai celebri Trucioli di Camillo Sbarbaro (Vallecchi, 1920), altro straordinario poeta e autore di aforismi purtroppo assai meno noto di quello che merita, soprattutto per i Fuochi fatui (Scheiwiller, 1956). Alla definizione concisa e unitaria viene preferita la moltiplicazione delle definizioni. Così è in Papini come in Bufalino. Ancora prima possiamo trovarne un esempio indicativo nel pensiero che apre il Centivio di Giuseppe Prezzolini:

Schizzi senza chiaroscuro, disegni e progetti restati sulla carta; domande senza risposta e risposte senza domanda; capitoli di romanzo, paragrafi di trattato, passi di libello; idee sbozzate, tentativi abbandonati, princìpi senza fine, programmi non eseguiti; saggi che non svolsi, prove mal riescite, germi troppo verdi, semi già secchi; affermazioni senza prove, deduzioni da premesse che mancano; punti interrogativi, lineette, ammirazioni, preludi, sinfonie, intermezzi; - ecco quel che contiene questo libro ${ }^{12}$.

Simile operazione accumulativa caratterizza il risvolto di copertina di Bluff di parole, la seconda raccolta di aforismi di Bufalino (Bompiani, 1994):

Da una più ampia miscellanea di citazioni, motti, macerie di diario, pensieri à gogo, Gesualdo Bufalino dichiarò d'aver tratto, anni addietro, le pagine edite sotto il titolo Il malpensante. Analoga origine possiede questa seconda puntata, incorniciata fra due lunari e divisa in capitoletti, non per ambizioni di architettura, bensì allo scopo di consentire piazzole di sosta e guanciali di spazi bianchi al lettore.

Al quale si consiglia, dunque, un assaggio parziale e vagabondo, come di chi spera di cogliere fra le gramigne del prato, non dico il semplice dalle miracolose virtù, ma qualche fungo mangereccio o, magari, un fiore da regalare.

L'elenco dovrebbe descrivere gli aforismi ma in realtà ne prolifica e galvanizza le interpretazioni con la citazione di opere del medesimo genere.

Nella quarta di copertina del Malpensante si aggiunge un elenco di autori «altissimi o alti, da Leopardi a Baudelaire, da Karl Kraus a Bierce, a Lec, a Flaiano». Di Leopardi si è già ampiamente detto. Di Baudelaire Bufalino ha amato gli aforismi e le riflessioni lapidarie di Fusées e di Mon cœur mis à nu. I restanti quattro autori rappresentano vertici della letteratura aforistica europea e americana. L'austriaco Karl Kraus, presentato sull'«Anima» di Papini e Amendola da Italo Tavolato nel 1911 e poi su «Lacerba» con una silloge di Aforismi nel secondo numero della rivista (15 gennaio 1913), è diventato familiare al pubblico italiano grazie all'edizione di Detti e contraddetti curata da Roberto Calasso nel 1972 per Adelphi (Bufalino possedeva la sesta edizione del 1987 e l'edizione Bur di Morale e criminalità del 1976). Kraus è senza dubbio l'aforista per eccellenza del 
Novecento, di «scorticante attualità» hanno scritto Fruttero e Lucentini («La Stampa Tuttolibri», 1 ottobre 1998). Il Dizionario del diavolo dello scrittore statunitense Ambrose Bierce, composto tra Otto e Novecento, fu tradotto in Italia a metà Novecento (Elmo, 1955; Bufalino possedeva l'edizione del 1956) e riproposto da Guido Almansi, altro cultore del genere aforistico, nel 1985 da Longanesi. È un modello di «comico nero», di cui offre un eloquente esempio Giuseppe Pontiggia nel volume aforistico Le sabbie immobili (il Mulino, 1991) con un'angolazione che sento vicina al pensiero di Bufalino:

Comico nero di Bierce

«Una mattina di buon'ora del giugno 1872 uccisi mio padre - un atto che, a quel

tempo, mi fece una profonda impressione» ${ }^{13}$.

Fra tanti mentitori edificanti, Bierce edifica l'unica cosa in cui crediamo quando abbiamo smesso di credere: la nostra distruzione.

E dobbiamo a scrittori solidali come lui se il male perde i contorni rassicuranti

dell'estraneo e acquista quelli più temibili di noi stessi ${ }^{14}$.

Di Stanisław Lec Bufalino aveva l'edizione dei Pensieri spettinati pubblicata da Bompiani nel 1984, curata da Pietro Marchesani e promossa da Umberto Eco, uno dei più convinti sostenitori dello scrittore polacco: «Se dovessi andare nella solita isola deserta e potessi portarmi dietro un solo libro, da leggere e rileggere, e la scelta fosse tra Kraus e Lec, io sceglierei Lec» ${ }^{15}$. Infine Flaiano, probabilmente il più malinconico e lucido dei nostri aforisti, con cui Bufalino condivide l'importanza dell'«errore» inteso come modo di pensare indipendente, non importa se giusto o sbagliato, conta che sia libero. Afferma Pickwick in un aforisma illuminante di Diario degli errori (Bompiani, 1956): «I posteri giudicheranno certo assai meglio le nostre questioni ma se noi non sbagliassimo sarebbe la fine. Sia ben chiaro che per saggezza intendo la capacità di agire in armonia coi miei errori preferiti»; e ancora questa osservazione dal postumo Diario degli errori, datata 1965: "Un altro anno ci lascia. Abbiamo vissuto commettendo errori, l'unico modo di vivere senza cadere. Vivere è una serie ininterrotta di errori, ognuno dei quali sostiene il precedente e si appoggia sul seguente. Finiti gli errori, finito tutto» ${ }^{16}$. Bufalino incalza: «Amo teneramente i miei errori, solo quelli amo di me...»; forse anche perché «Una verità è pericolosa quando non somiglia a un errore» ${ }^{17}$.

Bufalino torna sui modelli e i nomi propri nell'intervista di Massimo Onofri. Nel passaggio dedicato al Malpensante Onofri chiede: «Modelli, per questo libro?» Bufalino risponde: «Non saprei indicarne: troppo alti tutti, da Pascal a Nietzsche, a Schopenhauer. E ci sono poi i moralisti francesi, per i quali ogni precetto è un tassello dell'edificio morale, serve a una sostanziale filosofia del comportamento» ${ }^{18}$.

«Moralista imperfetto», come si definisce nella stessa intervista, Bufalino aggiunge altri modelli ai precedenti, «troppo alti tutti», nello stesso tempo allontanandoli e avvicinandoli, schernendosi per il confronto e l'accostamento impegnativo ed elogiativo eppure sentendosi, giustamente, della stessa famiglia. È il modo bifronte e ossimorico che lo distingue; egli accoglie invece di separare, include anziché scartare, concentra e non disperde («L'ossimoro non è una ridondanza ma una contrazione, non uno scialo ma un'economia»), patteggia con l'esistenza («Metà di me non sopporta l'altra e cerca alleati»; «Vi sono due razze di stupidi: quelli che credono a tutto e quelli che non credono a niente. Purtroppo io appartengo a entrambe») ${ }^{19}$. Egli preferisce «accarezzare i nodi dentro di sé, senza risolversi a tagliarli con un'energica scure». Il verbo accarezzare è spia di una peculiarità essenziale di Bufalino, che ama i propri rovelli, li coltiva, appunto li accarezza con compassione e simpatia, li abita e non vuole lasciarli, non intende reciderli, a differenza del «nodo gordiano» troncato dal colpo di 
spada di Alessandro Magno. Nodi gordiani è tra l'altro il titolo di un gruppo di trentasei aforismi che esce nello stesso 1987 sulla rivista «Nuovi argomenti» (gennaiomarzo 1987, pp. 65-67) e costituirà la prima sezione del successivo libro di aforismi Bluff di parole (Bompiani, 1994). Bufalino sceglie di convivere con i propri grovigli inestricabili, le ripetute e irrisolte interrogazioni, i punti di sospensione.

Superata la doppia soglia della copertina e della quarta di copertina entriamo dentro l'opera, ben sapendo però che essa è già cominciata. In esergo troviamo due citazioni lapidarie, la prima di Orazio («Male salsus homo»), la seconda di Pascal («Diseur de bons mots, mauvais caractère»). La prima citazione (Satire I, IX, 65) produrrà anche il neologismo bufaliniano «malesalso» equivalente a «spiritoso da strapazzo», come documentato dalla corrispondenza tra Bufalino e Gualberto Alvino ${ }^{20}$. In questo caso è però da sottolineare lo spirito maligno e agro della definizione, che scaturisce da una satira velenosa, in cui l'io narrante è preda di personaggi invidiosi e seccanti che non riesce a scrollarsi di dosso. L'attenzione va quindi posta sulla radice mal, che lo accomuna a malpensante e al successivo mauvais di Pascal.

La seconda (Pensées, 549) è una delle definizioni più note di 'bello spirito', caustico, aforistico, epigrammatico. Il titolo più celebre che la cita è Mauvaises pensées di Paul Valéry (José Corti, 1941 e Gallimard, 1942), altro sferzante capolavoro della letteratura aforistica del Novecento. L'aforisma è uno dei preferiti di Leonardo Sciascia, che in Nero su Nero lo riprende in due brani rilevanti. Nel primo per ribadire le proprie riserve nei confronti del saggio di Freud sui Motti di spirito e il loro rapporto con l'inconscio il cui risultato finale, "dopo un'analisi piuttosto faticante, si può dire che l'avevamo già a portata di mano nel "diseur de bons mots, mauvais caractère" dei francesi». La seconda è inserita in un partecipe e bellissimo ritratto di Ennio Flaiano, la cui satira verso la società del boom economico Sciascia paragona a quella compiuta da Brancati nei confronti del fascismo e dell'immediato dopoguerra. «Certo, Brancati dipendeva da Gogol. E Flaiano da Longanesi. Ma c'è, nelle cose di Flaiano, qualcosa di più - coscienza di sé, malinconia, pietà - che in quelle di Longanesi. Longanesi era il "diseur de bons mots" cui si accompagnava, secondo dicono i francesi e secondo ribadisce Freud nel suo saggio sui motti di spirito, un "mauvais caractère"». Invece "Flaiano non era un "mauvais caractère". E non si divertiva poi tanto, alle proprie battute»" Sciascia, Pascal, Flaiano sono uniti dall'ammirazione di Bufalino e da un'epigrafe densa di storia e di passione letteraria.

Sul piano aforistico Sciascia e Bufalino condividono il modello delle «battute apocrife», che Bufalino converte in un proprio Quaderno degli apocrifi. In Sciascia:

Battute (apocrife) tra due potenti, al momento dei saluti. Il primo, prolungando la stretta di mano: «Ah, dimenticavo... in Italia, naturalmente, le cose restano per come stanno». Il secondo, imprimendo alla stretta il vigore di una rinnovata e riassicurata lealtà: «Naturalmente». (Metternich stava a letto con una sua amante. E costei, a un certo punto: «E il povero principe di Monaco?». Metternich saltò giù dal letto e alla bozza del trattato aggiunse: «Il principe di Monaco rientrerà nei suoi Stati». La diplomazia, oggi, non ci concede né l'imprevedibile né il piccante ${ }^{22}$.

In Bufalino:

Dal Quaderno degli apocrifi.

«Preferendo ai caffè di Santippe una sostanziosa cicuta...» (Vita dei filosofi illustri)

«Mah!» (Sherlock Holmes sul letto di morte. Watson dixit)

«Inoltre l'acqua era tanta» (dai Racconti al bar d'un reduce dell'Invincibile Armata)

«Turirù totò titurera turù turututurù» (Charlie Parker, Relaxin' to Camarillo)

«Assoli d'olifante, telegrammi di poche parole...» (La Chanson de Roland Barthes) ${ }^{23}$. 
questo aforisma funziona anche il modello delle autocitazioni che Bufalino usa in più occasioni. Alcuni apocrifi erano comparsi nel racconto L'ingegnere di Babele, che prefigura l'idea di uno smisurato Dizionario di citazioni la cui composizione è quasi coincidente con quanto scritto nella quarta di copertina del Malpensante:

Un'impresa mirabolante, mi accorsi subito, inconfrontabile con i comuni repertori di aforismi che, attraverso stanche catene di plagi, si rubano a vicenda le stesse spiritose bugie; bensì, messa insieme a furia di letture eccentriche quanto mai, un'epitome certosinesca di incipit e desinit memorabili, un panopticon e bric-à-brac e scrapbook e merzbild e digest e miniera e mosaico e summa di motti, epigrafi, lampi, moralità, greguerías, agudezas, obiter dicta, disparates, poisons, fusées, mots-sésame, versi d'oro, foglietti della Sibilla... un incollaggio di schegge senza numero, sottratte occhiutamente a sotterranee Postumie e a solenni Partenoni, per essere offerte alla nostra impotenza in cambio delle desuete, ormai inconsumabili nozze con la polvere del passato ${ }^{24}$.

È un funambolico concerto di citazioni e allusioni, un divertimento enumerativo che fa esplodere in botti, razzi e lampi la classica terna su cui si fonda il movimento regolare dell'accumulazione. Nello stesso tempo Bufalino misura il significato delle parole che è sempre preciso e mai generico. Ogni parola ha un senso, lessicale, culturale, di genere, nella prospettiva in apparenza paradossale di un «barocco» che «vuol essere avaro»e «ambisce alle concentrazioni fulminee, pretende di inviare molti e sontuosi messaggi col minimo impiego di segni: come in quella convenzione licitativa del Bridge, che chiamano del "fiori napoletano" $»^{25}$.

La sagra delle citazioni non si esaurisce con le epigrafi d'apertura, che danno titolo e tono all'intero volume e sono il miglior autoritratto di Bufalino. Ognuna delle dodici sezioni del libro, corrispondenti ai mesi dell'anno, è introdotta da un'epigrafe. Le dodici del Malpensante sono di Foscolo, Paul-Jean Toulet, Juan Ramón Jimenéz, Geoffrey Chaucer, Tristan Corbière, Henry Thoreau, Gasparo Gozzi, Azorín (pseudonimo di José Martínez Ruiz), Madame de Sévigné, Jules Laforgue, Li Po, Emily Dickinson.

uff di parole il gioco si raddoppia. Il libro è diviso in due parti, Lunario dell'anno che viene e Lunario dell'anno che va, che sommate al precedente Lunario dell'anno che fu completano l'intero arco temporale di passato, presente e futuro. L'epigrafe iniziale è di Bufalino stesso, la poesia $\mathrm{Su}$ un calendario nuovo tratta da L'amaro miele. La novità strutturale è che le epigrafi dei mesi precedono e seguono il testo, che non ha più cadenza mensile ma è composto di sezioni che corrispondono a gruppi di aforismi già pubblicati su giornali e riviste. Le citazioni epigrafiche sono pertanto accorpate. Le dodici epigrafi di Lunario dell'anno che viene sono di Petrarca, Henry Thoreau, Emily Dickinson, Robert Frost, Leopardi, ancora Emily Dickinson, Marcel Proust, Hermann Melville, d'Annunzio, Stephane Mallarmé, Dante, François Villon. Lunario dell'anno che $v a$ è aperto da un'epigrafe di Alvaro de Campos (cioè Fernando Pessoa), la poesia $\mathrm{Nel}$ breve numero di dodici mesi. Le dodici dei mesi sono di James Thomson, Alceo, Anna Achmatova, Sainte-Beuve, Bondie Dietaiuti, Fazio degli Uberti, Nathaniel Hawthorne, Vincente Aleixandre, Gottfried Benn, Joseph Joubert, Madame de Sévigné, Wallace Stevens, Marina Cvetaeva, T. S. Eliot. Una panoramica sulla letteratura internazionale, antica e soprattutto contemporanea, di poesia più che di prosa, con due occorrenze per Thoreau, Leopardi e Madame de Sévigné, tre per l'amatissima Emily Dickinson.

Dopo copertina e quarta di copertina, dopo le epigrafi iniziali, si entra più propriamente nel testo, ben sapendo però che tutte le soglie introduttive sono già testo, secondo quest'altro aforisma del Malpensante:

Cahiers d'études italiennes, 30 | 2020 
Mi piace pensare a volte che i nomi degli scrittori che amo siano pseudonimi miei ${ }^{26}$.

È quanto scrive a proposito della traduzione delle greguerías di Ramón Gómez de la Serna (Bompiani, 1997):

Magro il mio spagnolo, scolastico il vocabolario di cui mi servo, ma bastevoli a fare emergere, parola dopo parola, con lo stesso sfizio che se ne fossi io l'autore, da quei brevi o minimi testi un'acutezza bizzarra, un'analogia strabiliante, uno scatto di elettrico umore ${ }^{27}$.

Gli aforismi di Bufalino sono strapieni di citazioni, esplicite e implicite. Sarebbe utilissimo fare un indice dei nomi e delle opere citate, certamente non solo per i libri di aforismi ma per tutti. Senza dubbio tale frequenza di citazioni in libri di aforismi è insolita e originale. Anche perché Bufalino mantiene quasi sempre la misura corta, lapidaria dell'aforisma, fedele (con libertà) all'idea che «Un aforisma benfatto sta tutto in otto parole» ${ }^{28}$.

Le citazioni possono servire da base e spunto per un commento, conferma di un'opinione, incontri e incroci di pensieri che attraversano il tempo:

Vi sono pensieri che non finiscono di viaggiare nei secoli. Pascal: «Il minimo movimento interessa tutta la natura: il mare intero cambia per una pietra». Leibniz: «L'universo è tutto d'un pezzo, come un oceano: il minimo movimento vi estende il proprio effetto a qualsiasi distanza...». Ebbene, se è così, non mi piace. Non voglio che questo granello d'infima sabbia ch'io sono abbia a turbare in nulla la macchina delle cose, io me ne lavo le mani.

Max Jacob (Le cornet à dés) raccontò d'aver incontrato un centauro sulle strade di Bretagna, D’Annunzio in Versilia. Altri ha visto per fiumi e mari orche, sirene... Ma io che dovrei dire di quella storia con Diana, mezzo secolo fa, laggiù, dove l'acqua dell'Ippari faceva gomito, sotto l'ombra verde d'un melo?

Quella contrerime di Toulet («Tempo è, Geronte, di scansar l'amore / e i suoi rabbiosi dardi. / Per sfidare onde e venti è troppo tardi / quando l'estate muore...») non vale solo per l'amore, vale anche per l'amicizia. Non fatevi amici nuovi, da vecchi.

La provincia: fodero, castello d'Atlante, prigione...?

«Come potrei vivere in un'aria che non fosse quella del carcere?» (Kafka).

Altre citazioni coincidono con l'aforisma stesso, affermazioni e interrogazioni dell'autore-pseudonimo:

«E se nella casa il ladro non c'è?». «Andremo nella casa accanto». «E se non c'è nessuna casa accanto?». «Ne costruiremo una» (Dialogo dei Marx Brothers).

«Nulla è più pericoloso di un'idea quando è l'unica che abbiamo» (Alain).

Alcune si distendono in liste:

Antiche lune. Il 26 dicembre 1814 Stendhal guardò la luna e gli parve una neve pestata da bestie dai piedi rotondi. Il 12 aprile 1834 Emerson ascoltò musica in un villaggio, sotto una bella luna gialla. Il 22 ottobre 1842 Nathaniel Hawthorne fece il bagno in un fiume ch'era calmo come la morte, e gli parve di tuffarsi nel cielo. Il 31 agosto 1849 Delacroix giocò a tombola in un giardino, al chiaro di luna, e ascoltò molte canzoni da un certo signor Bontemps. L'indomani si svegliò triste. Il 15 ottobre 1913 Kafka vide una carrozza fermarsi davanti a una casa e dalla finestra del quarto piano un giovane affacciarsi a guardarla, nel chiaro di luna... Dove siete, in che cimitero, care lune del tempo che fu?

I libri di mio padre: Ortis, Il fabbro del convento, La Divina Commedia (con le illustrazioni del Doré), Le veglie del Tasso (una contraffazione romantica che presi per buona), Il mistero del poeta, la Bibbia (quella protestante del Diodati), Col ferro e col fuoco, La pace e la guerra nel mondo antico (Ciccotti), I miserabili, Ti ha piaciato? (Petrolini), Guerra e pace, Manuale dell'artigliere, Quo vadis?, Letture geografiche (Marinelli), La bella argentiera, Marco Visconti, L'impiccato delle cascine... 
Sulla scia dei progetti di libri di Leopardi e anche di quelli di Barthes di Roland Barthes Bufalino propone «idee di racconto» sotto forma di citazione, dichiarata o sottintesa, anche in questi casi facendo equivalere aforisma e citazione:

«Due persone in attesa di un avvenimento e dei due principali attori di esso scoprono che l'avvenimento sta avendo luogo proprio allora e che i due attori sono essi stessi» (Hawthorne, Altro progetto, nei Diari, 25 settembre 1836).

L'esempio è citato pure da Borges nel saggio dedicato a Hawthorne in Otras Inquisiciones, «il cui tema (non ignorato da Pirandello o da André Gide) è la coincidenza oppure confusione del piano estetico e del piano comune, della realtà e dell'arte» ${ }^{29}$.

Ispirata invece a Oscar Wilde è la seguente idea narrativa:

Ipotesi di racconto: Collezionista di un solo quadro, lo appende al muro d'una

stanza vuota, ogni giorno va a guardarselo un'ora, lo distrugge prima di morire ${ }^{30}$.

Tra gli autori più citati c'è Joseph Joubert, uno dei giganti del pensiero aforistico. I suoi Carnets, da cui nel 1838 Chateaubriand ricavò una fortunatissima antologia di pensieri (Recueil des pensées de M. Joubert, Paris, Le Normant), sono un capolavoro assoluto. In Italia vi hanno dedicato meritoria attenzione Giuseppe Prezzolini, Corrado Rosso, Arnaldo Pizzorusso, Valerio Magrelli. Nel 1992 (il 21 settembre) Lalla Romano, in una intervista sul quotidiano «L'Unità», alla domanda quale libro «vorrebbe fosse ristampato» rispose:

Il Diario di Joseph Joubert uscito nell'Universale Einaudi nel 1943. È un libro che ho molto amato e che ho approfondito leggendone edizioni più complete in francese. Joubert è uno dei miei maestri di vita e di letteratura. C'è in lui un grande amore per la parola. Ricordo un suo pensiero, lo cito a memoria. «Mi piacerebbe che un libro si riducesse a una pagina, la pagina a una frase e la frase a una parola».

L'edizione ricordata da Lalla Romano è quella Einaudi 1943 curata da Mario Escobar. Bufalino possedeva l'ottima edizione delle Riflessioni pubblicate dall'editore Casini nel 1957 per la traduzione e la scelta di Guido Saba. Si tratta di cernite, più ampia la seconda della prima. Purtroppo tuttora non c'è in Italia un'edizione completa dei Carnets. La riproposta più recente è quella di Pensieri per vivere, a cura di Gavino Manca, pubblicata nel 2008 da Guerini e Associati.

Nel Malpensante Bufalino propone quattro aforismi ispirati a Joubert; in Bluff di parole è sua l'epigrafe di agosto di Lunario dell'anno che va:

Senza l'accompagnamento del canto della cicala, il tremolio dell'aria estiva, quando il sole brucia e il caldo è estremo, è una danza senza musica.

Joubert, Taccuini, 11 agosto $1813^{31}$

Le citazioni di Joubert proposte da Bufalino vanno in due direzioni: la prima è quella romantica, manifestata da un nuovo sentimento della natura:

Ameni boschi e cupe selve, fino al Seicento, apparivano sempre al passeggero come popoli d'alberi e comunità. Ci volle qualche secolo perché (1786) Joubert vedesse un pioppo solitario su una montagna e lo trovasse triste.

La seconda è quella satirica e politica, in cui Bufalino vede in Joubert possibili preavvisi di Kraus, soprattutto in chiave antigiornalistica:

La gazzetta che stamani pretende di aggiornarmi sullo stato delle cose com'è reticente, pettegola e favolosa, con quante balbuzienti verità m'imbonisce. «Mercanti di rumori», chiamava Joubert i giornalisti, ed era il 26 gennaio del 1823. In un secolo e mezzo non è cambiato granché, anzi il rumore è cresciuto e lo vendono più caro ${ }^{32}$. 
44 conoscenza diretta dell'autore, che induce Bufalino a spendersi con generosità per autori quasi sconosciuti. L'autore di cui voglio parlare è Carlo Gragnani, di cui Bufalino presenta alcuni aforismi in Bluff di parole, con questa introduzione: «Tempo fa un pensionato svizzero, italofono, di nome Carlo Grignani, mi spedì un manipolo di pensierini che meritano la luce. Senza chiedergli il permesso ma fiducioso di non dispiacergli, ne riferisco qualcuno $»^{33}$. In Bluff di parole scivola un refuso che poi è rimasto anche nelle successive edizioni: il nome esatto non è Grignani ma Gragnani. nella stessa via in cui abitava Prezzolini. Settantre anni, vedovo, ha alle spalle una prestigiosa carriera bancaria internazionale e un passato buddhista, con diversi articoli e monografie.

Dal punto di vista culturale è un appartato, un illustre sconosciuto. Una vita vissuta con discrezione, in cui la passione letteraria si è affacciata tardi e ha trovato propizia vena espressiva nelle forme brevi, soprattutto raccontini, apologhi, aforismi. La prima raccolta, A conti (quasi) fatti fu pubblicata a proprie spese nel 1989: esordiente alla soglia degli ottant'anni. Ne sono seguite diverse: Si fa per dire... (1992), In altri termini (1994), Scritti della domenica (1995), "Je est un autre» (1997), Non c’è altro (1998), Senza impegno (1999), A tempo perso (2000), Radici (2001), Buon natale (2002), Se son rose sfioriranno (2004), Ultimi arrivi (2005-2009). Testi che sono stati affettuosamente e autorevolmente riuniti e presentati dal filosofo Carlo Sini nei due volumi Per amor di completezza stampati a Lugano nel 2010, in occasione dei cent'anni dell'autore, deceduto pochi giorni prima di compierli. Poi nel 2012 Fabrizio Caramagna ha curato un'ampia scelta degli aforismi di Gragnani per le edizioni Genesi di Torino (Aforismi 1989-2010).

47 I primi libri di Gragnani mi furono spediti dall'autore proprio su indicazione di Bufalino, a cui devo perciò la scoperta di questo aforista arguto e penetrante che nel 1996 ho inserito nel secondo Meridiano Mondadori degli Scrittori italiani di aforismi dedicato al Novecento. Gli aforismi di Gragnani pubblicati da Bufalino in Bluff di parole sono in sintonia con i suoi temi preferiti:

«Je suis un autre», dice Rimbaud. Magari!

«Quando metterai giudizio?»

"Quando verrà il Giorno».

Il più grande sforzo della teologia è stato sempre quello di scagionare Dio.

Le sale d'attesa degli ospedali non indicano prudentemente che cosa dobbiamo

attenderci.

Il pessimista è uno che si è informato.

Il testo corretto del primo aforisma di Gragnani non è però "“Je suis un autre", dice Rimbaud. Magari!» ma «"Je est un autre" dice Rimbaud. Magari!» ${ }^{34}$, che riprende alla lettera la celeberrima autodefinizione presente nelle lettere a Georges Izambard del 13 maggio 1871 e a Paul Demeny del 15 maggio 1871 (quest'ultima nota anche come la Lettre du voyant). «Je suis l'autre» è invece un'affermazione di Gérard de Nerval posta sotto un proprio ritratto del 1854.

A Gragnani dedicò un bellissimo ritratto anche Giuseppe Pontiggia nell'Album di dicembre pubblicato sul supplemento culturale del «Sole 24 Ore» domenica 4 gennaio 1998. Il pezzo è datato « 31 dicembre», giorno emblematico perché sigla i calendari aforistici di capodanno. "Quando tenevo, anni fa, corsi di scrittura a Lugano», ricorda Pontiggia, 
[...] avevo notato tra i partecipanti un signore anziano, molto elegante e garbato, che faceva interventi calibrati, spesso ironici e corroboranti. Mi aveva anche mostrato certi suoi quaderni di aforismi e io li avevo tanto apprezzati da commentarli durante le lezioni. Da allora mi ha mandato a ogni fine anno, come in questo scorcio del '97, una piccola raccolta di aforismi, sulla quale gli ho sempre detto le mie impressioni. Mi sembrava un maestro in ombra di aforismi che meritavano la luce.

È stato grazie alla liberalità e all'intelligenza di Bufalino che questo «maestro in ombra» è venuto per virtù e per fortuna in luce.

\section{NOTE}

1. G. Ruozzi, Un malpensante fra libri, giochi e ricordi, «Studi e problemi di critica testuale», $\mathrm{n}^{\circ} 36$, aprile 1988, pp. 181-216.

2. G. Ruozzi, Forme brevi. Pensieri, massime e aforismi nel Novecento italiano, «Goliardica», 1992, pp. 429-481.

3. G. Bufalino, Il malpensante. Lunario dell'anno che fu, Milano, Bompiani, 1987.

4. E. Canetti, La provincia dell'uomo. Quaderni di appunti 1942-1972, trad. it. di F. Jesi, Milano, Adelphi, 1978, p. 193 (ed. orig., Die Provinz des Menschen. Aufzeichnungen 1942-1972, München, Hanser, 1978).

5. G. Bufalino, Il malpensante. Lunario dell'anno che fu, Milano, Bompiani, 1987, p. 118.

6. G. Bufalino, Il malpensante, cit., p. 139.

7. A. Cajumi, Pensieri di un libertino, presentazione di V. Santoli, Torino, Einaudi, 1970, pp. XIII-XIV, 290.

8. G. Bufalino, Nota a R. Gómez de la Serna, Sghiribizzi, scelta e traduzione di G. Bufalino, Milano, Bompiani, 1997, pp. 5-10 (la nota è datata 1995).

9. G. Papini, Le felicità dell'infelice, Firenze, Vallecchi, 1956, p. 3.

10. G. Papini, Un uomo finito, Milano, Mondadori, 1964, p. 77.

11. G. Bufalino, Il malpensante, cit., p. 93.

12. G. Prezzolini, Il Centivio, «Biblioteca del "Leonardo"», $n^{\circ} 4$, Milano, Libreria Editrice Lombarda, 1906, p. 7.

13. G. Pontiggia, Le sabbie immobili, Bologna, il Mulino, 1991, p. 53.

14. Ibid.

15. U. Eco, Sesamo apriti! Voglio uscire, in S. J. Lec, Pensieri spettinati, nuova edizione a cura di P. Marchesani, Milano, Bompiani, 1992, p. XII.

16. E. Flaiano, Diario notturno, in Opere 1947-1972, a cura di M. Corti e A. Longoni, Milano, Bompiani, 1990, p. 356; Diario degli errori, in Opere. Scritti postumi, a cura di M. Corti e A. Longoni, Milano, Bompiani, 1988, p. 367.

17. G. Bufalino, Il malpensante, cit., pp. 89, 104.

18. M. Onofri, Gesualdo Bufalino: autoritratto con personaggio (Comiso, 7 marzo 1992), in «Nuove Effemeridi», a. V, nº 18, 1992/II, p. 25 (ora in G. Bufalino, Opere/2 1989.1996, a cura e con introduzione di F. Caputo, Milano, Bompiani, 2007, p. 1333).

19. G. Bufalino, Il malpensante, cit., pp. 12, 16, 30.

20. G. Alvino, Gesualdo Bufalino (1920-1996) - Un epistolario, disponibile su <www.retididedalus.it>.

21. L. Sciascia, Nero su nero, Torino, Einaudi, 1979, pp. 42 e 127.

22. Ivi, p. 135. 
23. G. Bufalino, Il malpensante, cit., p. 84.

24. G. Bufalino, L'uomo invaso, in Opere 1981.1988, a cura di M. Corti e F. Caputo, introduzione di M. Corti, Milano, Bompiani, $2010^{2}$, p. 468.

25. G. Bufalino, Saldi d'autunno, in Opere/2 1989.1996, cit., p. 733.

26. G. Bufalino, Il malpensante, cit., p. 75.

27. G. Bufalino, Nota a Ramón Gómez de la Serna, Sghiribizzi, cit., p. 5.

28. G. Bufalino, Bluff di parole, cit., p. 86.

29. J. L. Borges, Altre inquisizioni, in Tutte le opere, a cura di D. Porzio, vol. I, Milano, Meridiani Mondadori, 2005, p. 959.

30. G. Bufalino, Il malpensante, cit., pp. 59, 77, 78, 76, 12, 77, 62 .

31. G. Bufalino, Bluff di parole, cit., pp. 115-116; J. Joubert, Riflessioni (Dai Diari), a cura di G. Saba, Roma, Casini, 1957, p. 326; Id., Carnets, textes recueillis sur les manuscrits autographes par A. Beaunier, avant-propos de J.-P. Corsetti, préface de Mme A. Beaunier et de M. A. Bellessort, 2 voll., Paris, Gallimard, 1994, vol. II, p. 393: «Sans l'accompagnement du chant de la cigale, le tremblottement de l'air en été, au grand soleil et dans le grande chaleur, est une danse sans musique».

32. G. Bufalino, Il malpensante, cit., pp. 35, 129; J. Joubert, Riflessioni, cit., pp. 17, 386 («Se vi è qualcosa di triste al mondo è il pioppo sulle montagne...»; «Mercanti di rumori, e li vendono: giornalisti, gazzettieri, ecc., ecc.»); Id., Carnets, cit., vol. I, p. 99 («S'il y a quelque chose de triste au monde, c'est le peuplier sur les montagnes...»); vol. II, p. 607 («Marchands de bruits, et qui le vendent : journalistes, nouvellistes, etc., etc.»).

33. G. Bufalino, Bluff di parole, cit., p. 58.

34. C. Gragnani, A conti (quasi) fatti, Milano, Cuem, 1989, p. 8; nel 1997 Gragnani utilizzò il «detto» di Rimbaud per il titolo di un nuovo libro di aforismi e forme brevi: «Je est un autre», stampato in proprio a Lugano.

\section{RIASSUNTI}

Scrittore coltissimo, Bufalino ha fatto frequente uso di citazioni sia come ornamento sia come ispirazione e confronto di idee. Struttura stilistica e retorica fondamentale di tutti i suoi testi, nei libri di aforismi essa diviene un modello specifico: l'aforisma citazione. Gli scrittori citati sono centinaia ma spiccano per sintonia Boiardo e Ariosto, Leopardi e Baudelaire, Joubert e Gómez de la Serna.

An extremely well-read writer, Bufalino made frequent use of quotations as embellishment but also as tools for comparison and sources of inspiration. Quotation is a basic stylistic and rhetorical structure present in all of his texts, but it becomes a specific trope in his books of aphorisms: "the quotation aphorism". Bufalino quotes hundreds of writers but feels particularly close to Boiardo and Ariosto, Leopardi and Baudelaire, Joubert and Gómez de la Serna.

Écrivain très cultivé, Bufalino a fait souvent usage de la citation, en tant qu'ornement mais aussi en tant que source d'inspiration et outil de comparaison. La citation est une structure stylistique et rhétorique présente dans tous ses textes, mais elle devient une forme spécifique dans ses livres d'aphorismes: «l'aphorisme citation». Bufalino cite des centaines d'écrivains, mais il ressent 
une affinité particulière avec certains d'entre eux : Boiardo et Ariosto, Leopardi et Baudelaire, Joubert et Gómez de la Serna.

\section{INDICE}

Mots-clés : citations, aphorismes, Boiardo, Ariosto, Leopardi, Baudelaire, Joubert, Gómez de la Serna

Parole chiave : citazioni, aforismi, Boiardo, Ariosto, Leopardi, Baudelaire, Joubert, Gómez de la Serna

Keywords : quotations, aphorisms, Boiardo, Ariosto, Leopardi, Baudelaire, Joubert, Gómez de la Serna

\section{AUTORE}

\section{GINO RUOZZI}

Università di Bologna 